\title{
CHARACTERIZATION OF WEAKLY IONIZED ARGON FLOWS FOR RADIO BLACKOUT MITIGATION EXPERIMENTS
}

\author{
L. Steffens, U. Koch, B. Esser, and A. Gülhan \\ German Aerospace Center (DLR) \\ Linder Höhe, Köln 51147, Germany
}

For reproducing the so-called $E \times B$ communication blackout mitigation scheme inside the L2K arc heated facility of the DLR in weakly ionized argon flows, a flat plate model has been equipped with a superconducting magnet, electrodes, and a setup comprising microwave plasma transmission spectroscopy (MPTS). A thorough characterization of the weakly ionized argon flow has been performed including the use of microwave interferometry (MWI), Langmuir probe measurements, Pitot probe profiles, and spectroscopic methods like diode laser absorption spectroscopy (DLAS) and emission spectroscopy.

\section{INTRODUCTION}

A reliable technique for solving the radio communication blackout problem during reentry is important for improving mission safety. Communication blackout occurs during reentry due to the ionization process of the surrounding shock layer, which creates a plasma layer. This plasma layer is not transparent for electromagnetic waves of a frequency below the plasma frequency thus blocking radio communications $[1-3]$.

For reproducing communication blackout in a ground based experiment, a flat plate model has been equipped with a setup comprising MPTS. This model setup has been tested inside the L2K arc heated facility of the DLR in weakly ionized argon flows. Argon flows have been used due to the reduced chemical complexity of the plasma to simplify comparison to numerical simulations. Suitable flow conditions for blackout mitigation tests have been identified by measuring the electron density by both MWI and MPTS.

The so-called $E \times B$ communication blackout mitigation scheme is based on reducing the electron density of the plasma layer locally above the antenna position by combined electric and magnetic fields. The combination avoids the need

(C) The authors, published by EDP Sciences. This is an Open Access article distributed under the terms of the Creative Commons Attribution License 4.0 (http://creativecommons.org/licenses/by/4.0/). 
of both strong magnetic and electric fields, therefore minimizing the required energy and improving Electromagnetic Compatibility (EMC) $[2,3]$.

A thorough characterization of the argon flow conditions has been performed to ensure a good comparability between experiments and numerical simulations. In particular, the plasma parameters - electron density and electron temperature - have been determined carefully by different techniques. The characterization is necessary for verification of numerical codes which are needed to transfer the blackout mitigation scheme to flight conditions. One main difference between flight application and the experiments in arc heated facility L $2 \mathrm{~K}$, is that in L2K the flow ionization is generated inside the arc heater whereas in flight case, it is caused by the heating in the shock layer of the reentry vehicle.

In the following, used flow conditions and methods for their characterization are presented and discussed. The model design for testing the $E \times B$ communication blackout mitigation scheme is described. Transmission spectra of the flow without applying the mitigation scheme are presented to verify that this experimental design is able to experimentally simulate the communication blackout. This is the first step of experimentally investigating the $E \times B$ blackout mitigation scheme. The principle design can be applied to other blackout mitigation schemes as well.

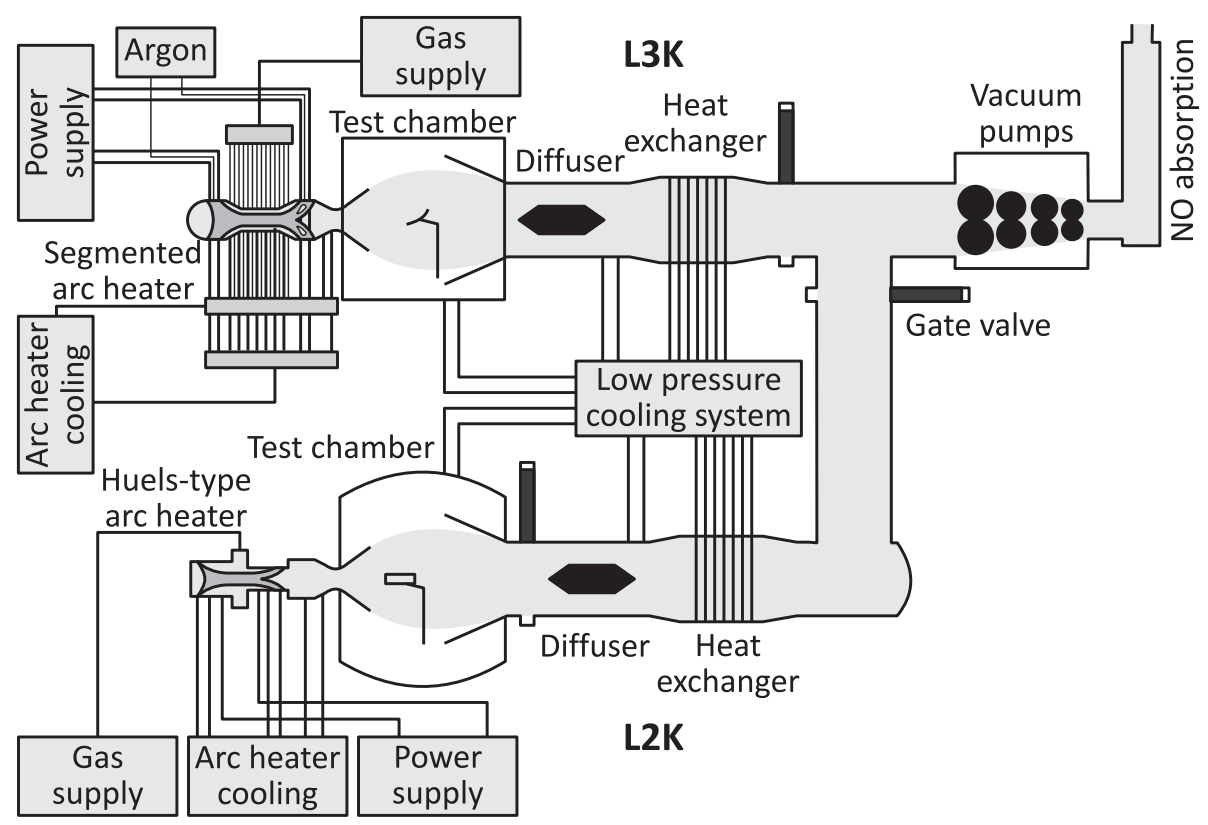

Figure 1 Arc heated facilities LBK of DLR 


\section{ARC HEATED FACILITY L2K}

For the tests, the L2K facility has been used. L2K is one of the test legs of DLR's arc heated facility LBK. A sketch of LBK is given in Fig. 1. The principal component of $\mathrm{L} 2 \mathrm{~K}$ is a Huels-type arc heater that has a maximum electrical power of 1.4 MW allowing to achieve cold wall heat fluxes in air flow up to $4 \mathrm{MW} / \mathrm{m}^{2}$ at stagnation pressures up to $250 \mathrm{hPa}$. Hypersonic free stream velocities are provided by a convergent-divergent nozzle. The nozzle's expansion part is conical with a half angle of $12^{\circ}$. Different throat diameters from 14 to $29 \mathrm{~mm}$ are available and can be combined with nozzle exit diameters of 50, 100, 200, and $300 \mathrm{~mm}$. So, the facility setup can effectively be adapted to particular necessities of a certain test campaign. A more detailed description of the L $2 \mathrm{~K}$ facility is given by Gülhan et al. [4-6].

\section{FLOW CHARACTERIZATION METHODS}

Coriolis gas mass flowmeter and reservoir pressure transducer have been used to continuously monitor the mass flow rate and the reservoir pressure during the tests. A Pitot probe has been applied for determining the Pitot pressure profile across the flow.

Emission spectroscopy with a Fourier transform infrared spectrometer (FTIR) has been used to study the species spectra and the electronic excitation state of both the free stream and within the reservoir chamber of the arc heater. For a summary of emission spectroscopy results, see [7,8]. Laser Induced Fluorescence Spectroscopy (LIF) on NO molecules seeded to the Argon has been used to determine the rotational temperature and concentration within the free stream and the bow shock layer $[9,10]$.

Diode laser absorption spectroscopy on carbon monoxide (CO) molecules has been used to determine the heavy particle translational temperature as well as the flow velocity of the free stream. Microwave interferometry has been used for line-of-sight determination of the electron density as well as determining the flow velocity. Different types of electrostatic probes have been used to estimate the electron temperature and electron density. Transmission spectra of microwaves have been used to determine the plasma frequency and, thus, the maximum electron density of the free stream. These four characterization techniques are discussed in detail in the following subsections.

\subsection{Diode Laser Absorption Spectroscopy}

Diode laser absorption spectroscopy was applied on CO molecules for measuring the flow velocity and the translational temperature using a diode laser emitting 
in the wavelength range from 2330 to $2335 \mathrm{~nm}$ at room temperature. Diode laser absorption spectroscopy is a line-of-sight method and the interpretation of the measurements has to take into account the homogeneity of the flow field. The velocity of the gas molecules is proportional to the spectral shift of the absorption signal and the translational temperature is determined from the half width of the absorption profile. In order to provide the required carbon monoxide for DLAS measurements, the argon flow was seeded with $20 \%$ of carbon dioxide which partially dissociates to carbon monoxide at high temperatures.

The measured velocity did not change significantly when the amount of seeding was changed from $5 \%$ to $40 \%$. For the measurement conditions in L $2 \mathrm{~K}$, the divergence of the flow direction tends to increase the extracted velocity value while shear layer effects in the outer region of the free stream tend to decrease

Table 1 Flow conditions

\begin{tabular}{|c|c|c|c|c|}
\hline Parameter & FC 1 & FC 2 & FC 3 & Comment \\
\hline Mass flow, $\mathrm{g} / \mathrm{s}$ & $20^{b}$ & $20^{b}$ & $20^{b}$ & \\
\hline $\begin{array}{l}\text { Reservoir } \\
\text { pressure, } \mathrm{hPa}\end{array}$ & $325^{b}$ & $350^{b}$ & $375^{b}$ & \\
\hline $\begin{array}{l}\text { Specific } \\
\text { enthalpy, MJ/kg }\end{array}$ & $1.5 \pm 0.1$ & $1.8 \pm 0.1$ & $2.0 \pm 0.1$ & NATA \\
\hline $\begin{array}{l}\text { Reservoir } \\
\text { temperature, } \mathrm{K}\end{array}$ & $2919 \pm 120$ & $3385 \pm 135$ & $3886 \pm 155$ & NATA \\
\hline Velocity, m/s & $\begin{array}{c}1723 \pm 9 \\
(-)^{a} \\
\end{array}$ & $\begin{array}{c}1850 \pm 30 \\
(-)^{a} \\
\end{array}$ & $\begin{array}{c}2150 \pm 73 \\
2350^{c} \\
\end{array}$ & $\begin{array}{l}\text { MWI } \\
\text { DLAS }\end{array}$ \\
\hline Temperature, K & $\begin{array}{l}(-)^{a} \\
(-)^{a}\end{array}$ & $\begin{array}{l}(-)^{a} \\
(-)^{a}\end{array}$ & $\begin{array}{c}180^{c} \\
80 \pm 3\end{array}$ & $\begin{array}{l}\text { DLAS } \\
\text { LIF }\end{array}$ \\
\hline $\begin{array}{l}\text { Pitot } \\
\text { pressure, } \mathrm{hPa}\end{array}$ & $4.5 \pm 0.1$ & $4.9 \pm 0.1$ & $5.6 \pm 0.1$ & Pitot \\
\hline Density, $\mathrm{kg} / \mathrm{m}^{3}$ & $1.52 \pm 0.02 \cdot 10^{-4}$ & $1.42 \pm 0.04 \cdot 10^{-4}$ & $1.25 \pm 0.08 \cdot 10^{-4}$ & \\
\hline $\begin{array}{l}\text { Electron } \\
\text { density, } 1 / \mathrm{m}^{3}\end{array}$ & $1.6 \pm 0.2 \cdot 10^{17}$ & $2.7 \pm 0.1 \cdot 10^{17}$ & $6.1 \pm 0.5 \cdot 10^{17}$ & MWI \\
\hline Ionization degree & $7.0 \pm 0.9 \cdot 10^{-5}$ & $1.3 \pm 0.6 \cdot 10^{-4}$ & $3.2 \pm 0.3 \cdot 10^{-4}$ & \\
\hline $\begin{array}{l}\text { Plasma } \\
\text { frequency } \omega_{p}, \mathrm{GHz}\end{array}$ & $23 \pm 1.4$ & $29 \pm 0.5$ & $44 \pm 1.8$ & \\
\hline $\begin{array}{l}\text { Plasma } \\
\text { frequency } f_{p}, \mathrm{GHz}\end{array}$ & $3.6 \pm 0.2$ & $4.7 \pm 0.1$ & $7.0 \pm 0.3$ & \\
\hline
\end{tabular}

${ }^{a}$ No DLAS and LIF measurements have been done for these conditions.

${ }^{b}$ Accuracy of $1 \%$.

${ }^{c}$ No accuracy considerations have been done. 
it. Concerning the temperature measurement, the broadening of the signal due to the shear layer leads to increased temperature values. As no quantitative information about the divergence of the flow vectors and the thickness of the shear layer is available, the presented data should be used for a qualitative interpretation of the flow properties [10]. The determined flow velocity and the translational temperature of all studied flow conditions are listed in Table 1 and discussed in section 4 .

\subsection{Microwave Interferometry}

Microwave interferomertry is used for measuring the electron density and the flow velocity of the free stream. An MWI 2650 microwave interferometer working at $26.5 \mathrm{GHz}$ measures the phase shift of microwaves passing the plasma. Electrons in the measurement volume reduce the permittivity and thus the optical path length on the way through the plasma. Thus, the phase shift is directly linked to the integral line of sight electron density in the measurement volume between emitter and detector. The effective geometrical path length is determined by Pitot probe profiles. The flow velocity is measured using two coupled MWI setups. An additional MWI 2650-V interferometer allows to determine the time fluctuations in the electron density need to travel with the flow from one interferometer arm to the other. By knowing the distance between the two measurement volumes, the flow velocity can be evaluated. The MWI 2650 is placed in 230-millimeter distance from the nozzle exit, the MWI 2650-V in 430millimeter distance. In contrast to the above described DLAS technique, no seeding of the flow is required.

The experimental setup of the MWI 2650 is sketched in Fig. 2. The measured electron densities and flow velocities for all studied flow conditions are listed in Table 1. A comparison with other methods for determining electron densities is given in subsection 4.2 .

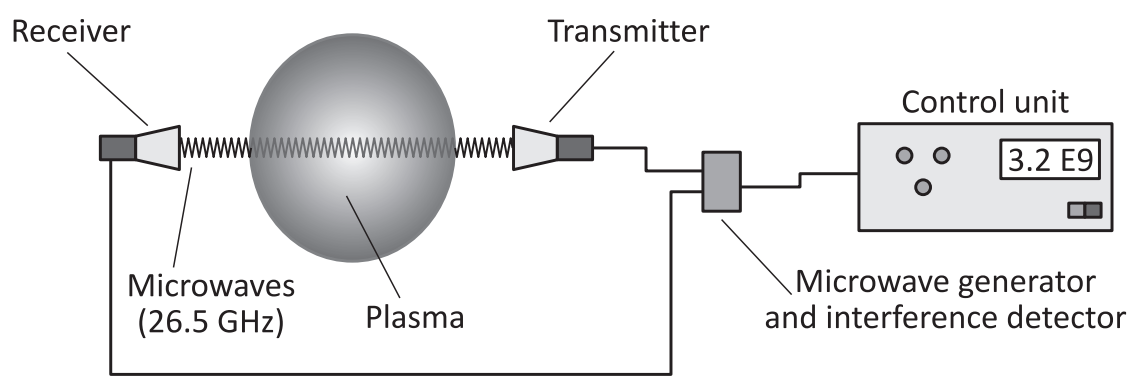

Figure 2 Scheme of MWI setup 


\subsection{Electrostatic Probes}

Langmuir probes in single [11], double [12], and triple configurations [13] were used to determine the electron density as well as the electron temperature of the flow. To exclude shock effects between the wires, the probe is placed behind the bow shock in the stagnation point region of a stagnation point model of 70-millimeter diameter. A photograph of the Langmuir triple probe setup in Argon flow is given in Fig. 3. In this picture, the bow shock in front of the triple wire arrangement is clearly visible. The electron temperature is supposed to be effected only by a small amount by the shock due to the higher speed of sound of the electron gas, so that

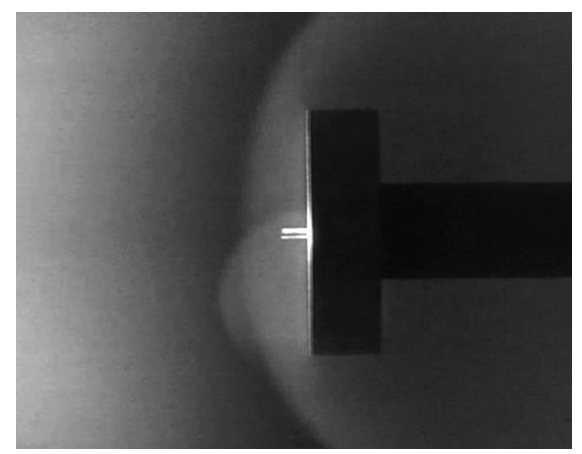

Figure 3 Langmuir triple probe in Argon flow the free stream electron temperature can be approximated by the measured value. In contrast, the electron density is supposed to be effected by the bow shock, which has to be taken into account when comparing it to the results of other methods. During the single and double probe measurements, the probes were placed on the flow axis. With the triple probe, also, density profiles across the flow have been recorded.

\subsection{Microwave Plasma Transmission Spectroscopy}

Radio transmission is the essential test parameter for blackout mitigation. To examine the radio blackout, a compact sender antenna is integrated within a flat plate model. The microwaves are transmitted through the quartz surface of the model and half the beam width of the L2K weakly ionized argon flow. They are received by an antenna placed within the $\mathrm{L} 2 \mathrm{~K}$ chamber outside the flow. A spectrum analyzer with a tracking generator measures spectra of the received signal strength. The transmission of the argon flow is determined by subtracting received spectra recorded without flow from those with flow. The setup is sketched in Fig. 4.

The sender antenna is a cavity backed spiral antenna with a diameter of $55 \mathrm{~mm}$ and a height of $50 \mathrm{~mm}$. A conical log spiral antenna is used as receiver antenna. The spectrum analyzer is a Rohde \& Schwarz FSL 18. The output signal of the spectrum analyzer has been amplified external amplifiers 


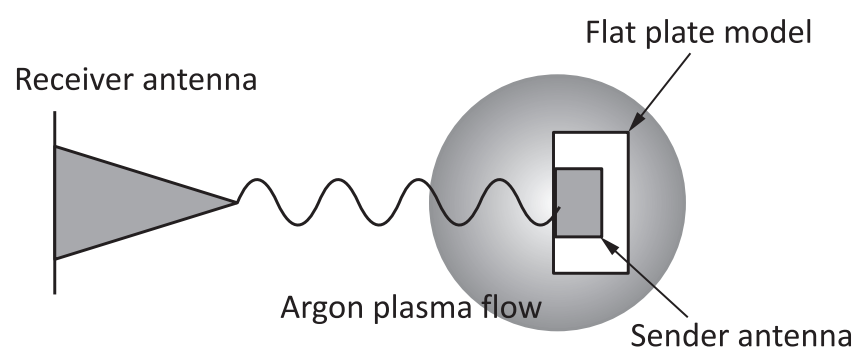

Figure 4 Sketch of MPTS setup

by an additional $20 \mathrm{~dB}$. This setup is able to record spectra in a range from 2 to $10 \mathrm{GHz}$. The noise level of the setup is at $-80 \mathrm{~dB}$.

The antenna polarization is circular polarized, as the L2K chamber has a metallic surface which is highly reflective for radio waves. Reflection of the waves changes the handedness of the circular polarization. So, the receiver antenna is much less sensitive to single time reflected waves, suppressing signal interferences. A set of both LHCP (left-hand circular polarization) and RHCP (right-hand circular polarization) antennas is available.

When the flow plasma frequencies are in the frequency range of the MPTS setup, it is expected to observe a rise in the transmission to about $0 \mathrm{~dB}$ at a distinct edge frequency which is linked to the maximum electron density $n_{e}$ of the transmission volume. By using

$$
\omega_{p}=\sqrt{\frac{n_{e} e^{2}}{m_{e} \varepsilon_{e}}}
$$

where $n_{e}$ is the electron density; $e$ is the elementary charge; $m_{e}$ is the electron mass; and $\varepsilon_{e}$ is the electric constant, and

$$
f_{p}=\frac{1}{2 \pi} \omega_{p},
$$

the maximum electron density $n_{e}$ of the flow is derived. The edge frequency is determined by the intersection of the 0 -decibel line and a linear extrapolation to the attenuation curve in the region of its largest slope. The obtained results for the edge frequency and the corresponding electron density $n_{e}$ are listed in Table 2 for all three conditions.

The (angular) plasma frequency $\omega_{p}$ is defined by Eq. (1). Also, in this paper the (ordinary) plasma frequency $f_{p}$ defined by Eq. (2) is used. 


\section{ARGON FLOW CONDITIONS FOR BLACKOUT MITIGATION TESTS AT L2K}

To fit the scale of the used flat plate models, the 200-millimeter nozzle exit diameter has been used for the blackout mitigation test. The previously studied argon flow condition FC 3 defined by a mass flow rate of $20 \mathrm{~g} / \mathrm{s}$ and a reservoir pressure of 375 mbar is used [9]. Two additional conditions FC 1 and FC 2 were defined using a mass flow rate at $20 \mathrm{~g} / \mathrm{s}$ and reducing the reservoir pressure to 325 and 350 mbar, respectively, resulting in a reduction in the enthalpy and, thus, the electron density. These three states feature electron densities in the measuring range of all used electron density measurement techniques and, also, give the possibility to determine the effectiveness of blackout mitigation techniques for different electron densities.

\subsection{Definition of Flow Conditions}

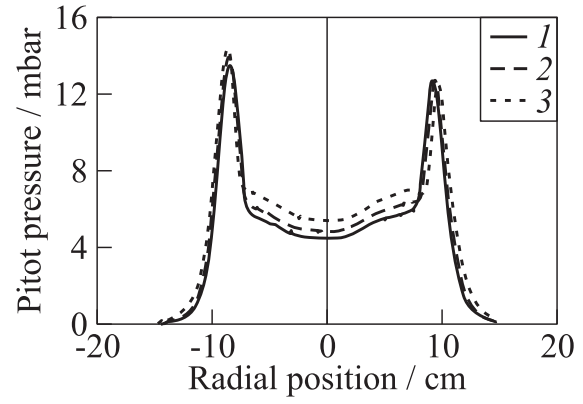

Figure 5 Pitot pressure profiles for FC 1 (1), FC 2 (2), and FC 3 (3)
The characterization results for each of the three conditions are listed in Table 1. From mass flow and reservoir pressure, the specific enthalpy and total temperature were computed using the NATA code [14], which is a quasi-one-dimensional flow solver including nonequilibrium chemistry. All other values are free stream measurement results taken at 330-millimeter distance from nozzle exit, measured by the given method. The MWI measured results of the electron density are taken at 230-millimeter distance from nozzle exit. The Pitot pressure profiles across the flow are given in Fig. 5. The pressure peaks at each side are caused by side shocks. The Pitot pressure value listed in Table 1 is the on-axis value. The densities are determined from velocity and Pitot pressure and plasma frequencies are derived using Eq. (1). The Pitot pressure profiles are used to derive density and the beam width, which is needed for getting the average electron density $n_{e}$ from the line-of-sight electron density determined by the MWI. No DLAS and LIF measurements have been done for FC 1 and FC 2 so far.

\subsection{Electron Density and Electron Temperature Measurements}

The values for the electron density $n_{e}$ obtained by MWI, MPTS, and Langmuir double probe $(2 \mathrm{~L})$ are compared in Table 2 . The determination of the MPTS 
Table 2 Comparison of $n_{e}\left(\mathrm{~m}^{-3}\right)$ measurement results

\begin{tabular}{lcccr}
\hline Condition & MWI & \multicolumn{2}{c}{ MPTS } & \multicolumn{2}{c}{ L } \\
\hline FC 3 & $6.1 \pm 0.5 \cdot 10^{17}$ & $5.6 \pm 0.4 \cdot 10^{17}$ & $(6.7 \pm 0.2 \mathrm{GHz})$ & $13.3 \pm 0.9 \cdot 10^{17}$ \\
FC 2 & $2.7 \pm 0.1 \cdot 10^{17}$ & $3.7 \pm 0.1 \cdot 10^{17}$ & $(5.5 \pm 0.1 \mathrm{GHz})$ & $7.0 \pm 0.4 \cdot 10^{17}$ \\
FC 1 & $1.6 \pm 0.2 \cdot 10^{17}$ & $1.7 \pm 0.1 \cdot 10^{17}$ & $(3.7 \pm 0.1 \mathrm{GHz})$ & $3.3 \pm 0.2 \cdot 10^{17}$ \\
\hline
\end{tabular}

Table 3 Comparison of the $T_{e}(\mathrm{~K})$ measurement results of different Langmuir probes

\begin{tabular}{lccc}
\hline Condition & 1L & $2 \mathrm{~L}$ & $3 \mathrm{~L}$ \\
\hline FC 3 & $3052 \pm 550$ & $1492 \pm 550$ & $2405 \pm 100$ \\
FC 2 & $2884 \pm 400$ & $1401 \pm 320$ & $(-)^{a}$ \\
FC 1 & $3583 \pm 900$ & $1502 \pm 315$ & $(-)^{a}$ \\
\hline \multicolumn{4}{c}{ Too few data points for this condition were taken to give a reli- } \\
able value.
\end{tabular}

values is described and discussed in detail in subsection 5.2. The Langmuir probe values have been determined by taking average values over several test runs. The results of the MWI and MPTS are in good agreement for all states. The values for the Langmuir double probe (2L) are about a factor of 2 higher than those for the other methods. A higher value has to be expected according to the placement of the probe wires behind the bow shock.

The results for the electron temperature $T_{e}$ of Langmuir single probe (1L), double probe $(2 \mathrm{~L})$, and triple probe $(3 \mathrm{~L})$ are compared in Table 3 . The given values have been determined by taking average values over several test runs. The data points marked with (-) indicate those conditions for which not enough data were obtained to get a reliable value.

Within the range of uncertainties, the electron temperature values gained by one method do not change with the flow condition. The values of single and triple probes are both in the range of 2500 to $3500 \mathrm{~K}$, whereas the values for the double probes are about a factor of 2 lower in the range of $1500 \mathrm{~K}$.

These two different but reproducible temperature values for the different types of probes are a hint that the assumptions for either one or both evaluation routines for the current voltage curves resulting in temperature values are not valid. Further research has to be done to clarify these deviations.

Electron density profiles across the flow measured by Langmuir triple probe are shown in Fig. 6. The steps in the profile are due to the slow current data acquisition compared to the voltage data acquisition. The values obtained by the triple probe measurements are in the range of the values obtained by MWI and MPTS. They have larger uncertainties than the other values due to the spread in the corresponding electron temperatures. 


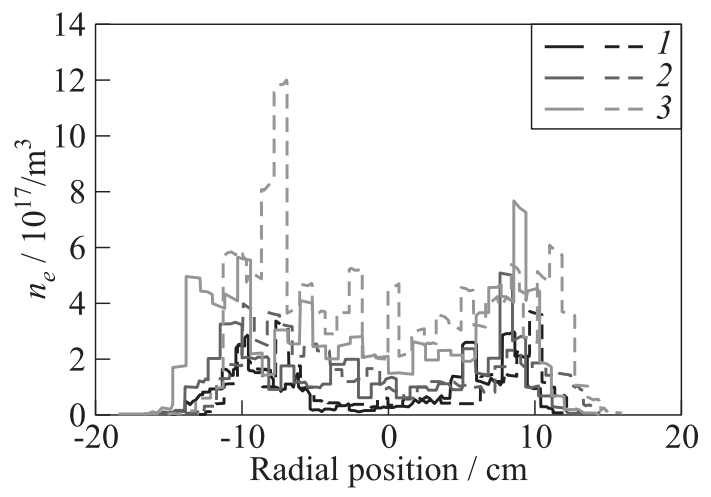

Figure 6 Electron density profiles measured by Langmuir triple probe: 1 - FC 1; 2 - FC 2; and 3 - FC 3. Solid lines refer to back sweeps through the flow and dashed lines to the forth ones

Further studies have to be done on the reliability of the values and the validity of using the standard analysis routines for determining the electron temperatures and densities from the obtained data.

\section{COMMUNICATION BLACKOUT EXPERIMENTS}

\subsection{Flat Plate Model for Testing $E \times B$ Communication Blackout Mitigation Scheme}

For testing the $E \times B$ communication blackout mitigation scheme, a flat plate model has been constructed comprising a high-temperature superconducting (HTS) magnet. The magnet and its cryogenic system have been constructed by the Institute for Technical Physics (ITEP) of the Karlsruhe Institute of Technology (KIT). The cryogenic system is able to cool the magnet down to temperatures of about $8 \mathrm{~K}$. The accessible magnetic field strength at the model surface is about 2 T. The setup is shown in Fig. 7. The cryostat is mounted in the L2K chamber together with the flat plat model. The magnet gets into the model by an opening in the model ground plate. The cryostat is protected from the flow and thus from high heat fluxes by a protective plate.

The flat plate model is shown in detail in Fig. 8. The magnet is placed beneath a 1-millimeter quartz glass plate in the center of the model. Two electrodes for generating the electric field of the $E \times B$ scheme are integrated into the model. The anode is placed between the model nose and the quartz plate. The cathode is placed at the rear end of the model. The compact sender an- 


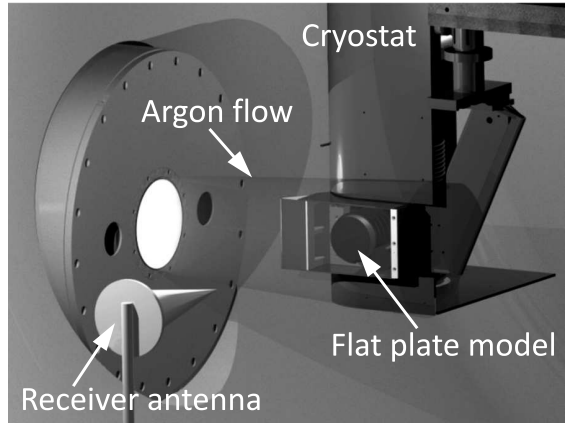

Figure 7 Sketch of experimental setup in $\mathrm{L} 2 \mathrm{~K}$ chamber

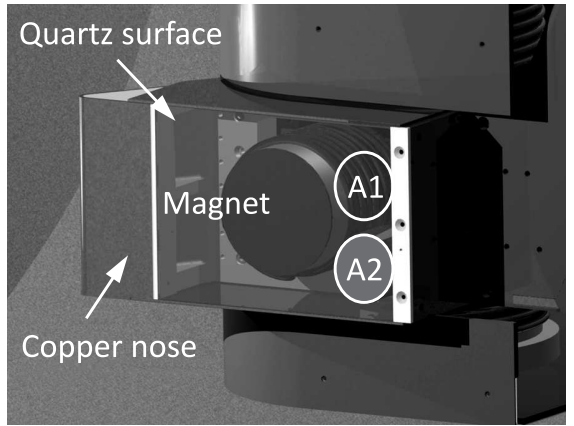

Figure 8 Sketch of flat plate model

tenna can be placed at two positions A1 and A2 just behind the magnet. In the following, only the upper antenna position A1 is used. The axis of the receiver antenna is aligned to the axis of the sender antenna. Its distance to the model is $30 \mathrm{~cm}$. The nose of the model is made from copper and cooled by water.

This setup in contrast to usual L2K models cannot be moved within the L2K chamber and it is directly mounted on the flow axis with a 0 degree angle of attack. As the HTS magnet is sensitive to high heat loads, the test runs are performed only for a few seconds after arc heater ignition.

\subsection{Measurement of Transmission Spectra Without Applying the Mitigation Scheme}

For experimentally reproducing communication, blackout transmission spectra of each previously specified flow conditions are recorded without any flow manipulation by $E \times B$ fields.

For a test, the cryostat is cooled down. The argon mass flow rate is set to $20 \mathrm{~g} / \mathrm{s}$. Then, a continuous recording of spectra with a rate of 3 to 4 spectra per second is started. After about $10 \mathrm{~s}$, the arc heater is ignited. The flow reaches a steady state with the specifications listed in Table 1 within about $10 \mathrm{~s}$. The test run is automatically aborted after $20 \mathrm{~s}$ by switching off the arc heater, resulting in about $10 \mathrm{~s}$ of steady state flow. The recording of spectra is continued for about $10 \mathrm{~s}$ after end. From this, one gets two sets of reference spectra without flow and one set of spectra with flow.

From these data sets, an averaged transmission spectrum for the respective flow condition is determined. First, all recorded spectra have been filtered with 


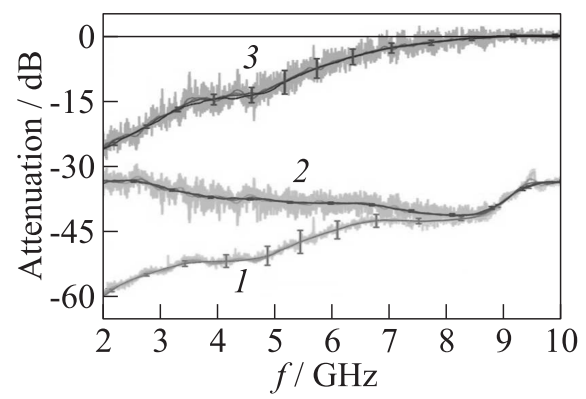

Figure 9 Determination of a transmission spectrum: 1 - attenuation with flow; 2 attenuation without flow; and 3 - transmission a moving average of a specific frequency width. This moving average filter reduces noise and remaining interference patterns. Afterwards, all filtered reference spectra and all filtered flow spectra are being averaged frequency-wise, resulting in a timeaveraged reference spectrum and a time-averaged flow spectrum. The reference spectrum is subtracted from the flow spectrum to get the time-averaged transmission spectrum. For plotting the spectra, a moving average of a width of $800 \mathrm{MHz}$ is used.

The determination of a transmission spectrum is depicted in Fig. 9. Curves 1 show the spectra with argon flow. Curves 2 show the reference spectra without argon flow. Curves 3 show the resulting transmission spectra. For each of these spectral sets, different moving averages with widths of 4, 200, 400, 600, and $800 \mathrm{MHz}$ are shown. The error bars show the respective standard deviation of the curves filtered with a width of $800 \mathrm{MHz}$.

Figure 10 shows all recorded spectra for conditions FC 1 to FC 3 with both the LCHP (solid lines) and RHCP (dashed lines) antenna setup for antenna position A1. The FC 1 tests are depicted by curves 1, FC 2 tests by curves 2 , and FC 3 tests by curves 3 . The error bars show an exemplary standard deviation of one test for each flow condition.

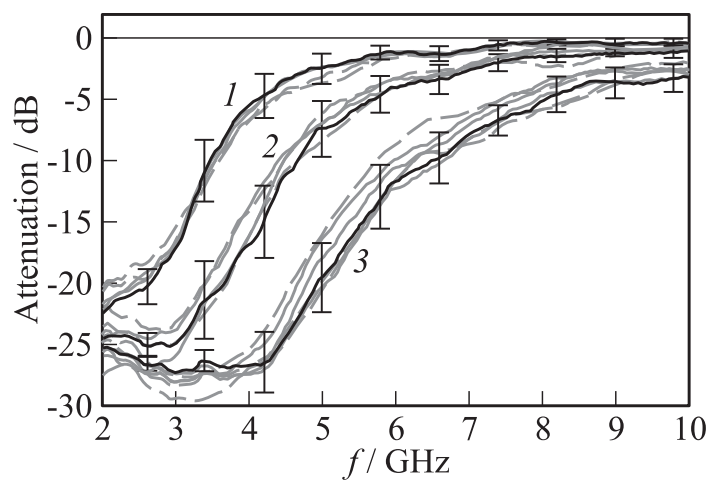

Figure 10 Attenuation spectra measured with LHCP (solid lines) and RHCP (dashed lines) antennas: $1-$ FC $1 ; 2-$ FC 2 ; and $3-$ FC 3 
The different flow conditions show clearly distinct attenuation curves. The spectra for one condition are comparable within standard deviation. There is no significant difference between the antenna setups. The attenuation has a level between $-20 \mathrm{~dB}(\mathrm{FC} 1)$ and $-28 \mathrm{~dB}(\mathrm{FC} 3)$ at the low end of $2 \mathrm{GHz}$. At a specific edge frequency, the transmission rises to a level between almost $1 \mathrm{~dB}$ (FC 1) and $3 \mathrm{~dB}(\mathrm{FC} 3)$ at the high end of $10 \mathrm{GHz}$.

For each spectrum, the edge frequency is evaluated by determining the intersection of the $0 \mathrm{~dB}$ axis with a linear extrapolation at the maximum slope. This edge frequency can be considered to be linked to the maximum electron density $n_{e}$ of the flow and its corresponding plasma frequency $f_{p}$. The average edge frequency for each condition is given in Table 2. Corresponding electron densities $n_{e}$ are calculated using Eq. (1). These are also listed in Table 2. The resulting values are in good agreement to the values determined by the MWI free stream. As an angle of attack of $0^{\circ}$ is used, the flow above the flat plate surface outside the boundary layer is expected to be similar to the free stream flow.

As expected, the plasma flow is transparent for microwaves above a specific frequency and microwaves are blocked beneath this frequency. Thus, the principle of radio communication blackout can be experimentally reproduced in L2K facility for the selected argon flow conditions. As additionally expected, the edge frequency is growing with the specific enthalpy of the flow. In conclusion, this experimental design is a promising basis for testing blackout mitigation schemes as the $E \times B$ scheme, for which the previously described HTS model is designed.

\section{CONCLUDING REMARKS}

Different argon flow conditions have been defined for experimental blackout mitigation simulation tests. A thorough characterization of those conditions has been done with multiple methods including spectroscopic methods and different methods to determine the electron density as well as the electron temperature.

For studying the $E \times B$ blackout mitigation scheme, a flat plate model setup comprising a superconducting magnet has been constructed. With this setup, the radio communication blackout can be experimentally reproduced in L $2 \mathrm{~K}$ facility for the selected argon flow conditions. From the resulting transmission, spectra electron densities could be estimated which are in good agreement with the electron densities measured by MWI. Thus, this principle experimental design is a promising basis for testing the $E \times B$ blackout mitigation scheme, in particular, and other blackout mitigation schemes, in general.

\section{REFERENCES}

1. Heald, M. A., and C. B. Wharton. 1965. Plasma diagnostics with microwaves. London, U.K.: John Wiley \& Sons. 452 p. 
2. Kim, M., M. Keidar, and I. D. Boyd. 2008. Effectiveness of an electromagnetic mitigation scheme for reentry telemetry through plasma. AIAA Paper No. 20081394.

3. Kim, M. 2009. Electromagnetic manipulation of plasma layer for re-entry blackout mitigation. University of Michigan. PhD Thesis.

4. Gülhan, A., and B. Esser. 2001. A study on heat flux measurements in high enthalpy flows. AIAA Paper No. 2001-3011.

5. Gülhan, A., and B. Esser. 2002. Arc-heated facilities as a tool to study aerothermodynamic problems of reentry vehicles. Advanced hypersonic test facilities. Eds. D. Marren and F. Lu. Progress in astronautics and aeronautics ser. Reston, VA: AIAA. 198:375-403.

6. Gülhan, A., B. Esser, U. Koch, and K. Hannemann. 2002. Mars entry simulation in the arc heated facility L2K. 4th European Symposium on Aerothermodynamics for Space Vehicles, Proceedings. Noordwijk, The Netherland: ESA Publications Div. ESA-SP-487. 665-772.

7. Koch, U., L. Steffens, B. Esser, A. Gülhan, and K. Shibusawa. 2014. Characterization of Argon flows in the arc heated facility L2K using Diode Laser Absorption spectroscopy on Argon and additional optical diagnostics. 6th Workshop (International) on Radiation of High Temperature Gases in Atmospheric Entry Proceedings. St. Andrews, UK. 10 p.

8. Shibusawa, K., L. Steffens, and U. Koch. 2014. Infrared spectroscopic measurements of argon plasma flow in arc heated facility L2K. 46th FDC/ANSS 2014 (Conference CD-ROM) Proceedings. JSASS-2014-2014-A. 1-7.

9. Koch, U., J. Riehmer, B. Esser, and A. Gülhan. 2009. Hypersonic free stream characterization in LBK by laser induced fluorescence and diode laser absorption spectroscopy. 3rd Workshop (International) on Radiation of High Temperature Gases in Atmospheric Entry Proceedings. Noordwijk, The Netherlands: ESA Publications Div. ESA-SP-667.

10. Gülhan, A., B. Esser, U. Koch, F. Siebe, J. Riehmer, D. Giordano, and D. Konigorski. 2009. Experimental verification of heat-flux mitigation by electromagnetic fields in partially-ionized-argon flows. J. Spacecr. Rockets 46(2):274-283.

11. Langmuir, I., and H.M. Mott-Smith. 1926. The theory of collectors in gaseous discharges. Phys. Rev. 28:727-763.

12. Johnson, E. O., and L. Maltereca. 1950. A floating double probe method for measurements in gas discharges. Phys. Rev. 80(1):58-68.

13. Chen, S. L., and T. Sekiguchi. 1965. Instantaneous direct-display system of plasma parameters by means of triple probe. J. Appl. Phys. 36(8).

14. Bade, W.L., and J. M. Yos. 1975. The NATA code, theory and analysis. NASA CR-2547. 